\title{
Septic thrombophlebitis of the sigmoid sinus after vestibular schwannoma resection: A case report and literature review
}

\author{
Thiago Pires Brito, Henrique Furlan Pauna*, Renato Caleffi Pereira, Raimundo Vinicius de Araújo Rego, Arthur Menino Castilho and Jorge \\ Rizzato Paschoal
}

Department of Otorhinolaryngology, Head and Neck Surgery, University of Campinas (UNICAMP), Campinas, São Paulo, Brazil

\begin{abstract}
Introduction: The translabyrinthine approach has been increasingly used in the removal of tumors of the cerebellopontine angle. Sigmoid sinus thrombophlebitis (SST) is a possible complication of this surgical approach.

Case report: Male, 69 years-old with progressive unilateral hearing loss on his left side, which started three years before, and tinnitus. Audiometry showed deafness in the left ear. MRI showed a $1.5 \mathrm{~cm}$ lesion in the left cerebellopontine angle. Surgical resection via translabyrinthine. Biopsy revealed vestibular schwannoma (VS). On the 15th postoperative day, he developed headache, blurred vision, dizziness, nausea and fever, no signs of meningeal irritation or other neurological deficits. The wound showed signs of inflammation. Imaging studies showed elongated filling defect extending from the transverse sinus to the sigmoid sinus on the left. He underwent intravenous antibiotic treatment, with good clinical evolution.

Discussion: The SST is a rare complication in the resection of VS. Among the possible explanations of the event stand out within retraction intraoperatively; sinus dissection during resection of the tumor and the spread of infectious processes from the mastoid causing septic thrombophlebitis. On contrast-enhanced CT can find central triangular area does not enhance and is bounded by the dura mater captive contrast, also known as a sign of "empty delta". Treatment may include anticoagulation, antibiotic therapy and surgical procedures.
\end{abstract}

Considerations: The incidence of SST decreased after intravenous antibiotic treatment. Diagnosis requires clinical suspicion and imaging studies.

\section{Introduction}

Inflammation and infection of the middle ear and mastoid air cells predispose to thrombophlebitis of the dura mater venous sinuses due to the proximity between these structures [1]. Thrombosis and thrombophlebitis of these vessels are relatively uncommon but potentially fatal if underdiagnosed [2]. With the advancement of imaging techniques in the last decades, it was possible to increase the number of diagnoses and to know more about this pathology [1-3].

Cerebral venous sinus thrombosis is associated with several clinical conditions such as thrombophlebitis, trauma, dehydration, hematological alterations and otologic/intracranial surgeries. In the case of thrombophlebitis, the main etiology is infectious, and, in this case, it is called septic thrombosis of the sigmoid sinus [4-7].

Since the introduction of translabyrinthine access in 1960, this pathway has become increasingly popular for the removal of neoplasms from the cerebellopontine angle [1].

We report the case of a patient who presented sigmoid sinus thrombophlebitis (SST) after resection of vestibular schwannoma (VS) and review the literature.

\section{Case report}

A 69-years-old male patient attended at the Otorhinolaryngology outpatient clinic of our Institution with left hearing loss during the past three years, progressive and associated with tinnitus. As a personal background, hypertension and diabetes were poorly controlled. No other otorhinolaryngological complaints.

During investigation, the audiometry showed profound hearing loss in the left ear and normal auditory thresholds in the right ear. A MRI scan was obtained and showed a $1.5 \mathrm{~cm}$ lesion in the left cerebellopontine angle compatible with vestibular schwannoma (Figure 1). CT scan showed a lesion at the same location, isoattenuating to the cortex and homogenous contrast enhancement (Figure 2).

He underwent surgical resection of the lesion via the translabyrinthine approach, without trans-operative complications. In the immediate postoperative period, he presented grade IV facial paralysis. He was discharged from hospital on the $4^{\text {th }}$ postoperative day in good clinical condition, biopsy confirmed that it was schwannoma (Figure 3).

${ }^{*}$ Correspondence to: Henrique Furlan Pauna, Department of Otorhinolaryngology, Head and Neck Surgery, University of Campinas (UNICAMP), Campinas, São Paulo, Brazil, E-mail: h_pauna@hotmail.com

Key words: thrombophlebitis, mastoiditis, sinus thrombosis, schwann cells, acoustic neuroma

Received: April 13, 2018; Accepted: May 22, 2018; Published: May 31, 2018 


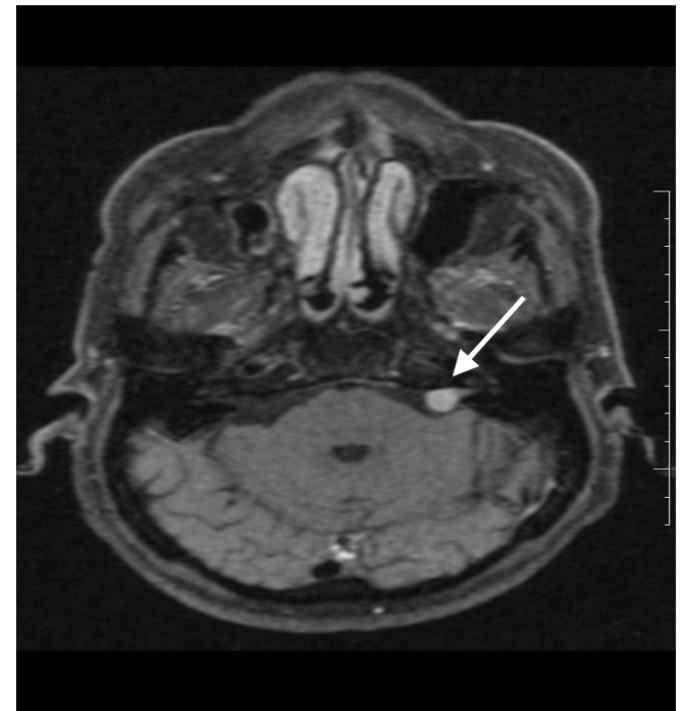

Figure 1: The first author wearing an anterior-half body cast fixed with a soft brace
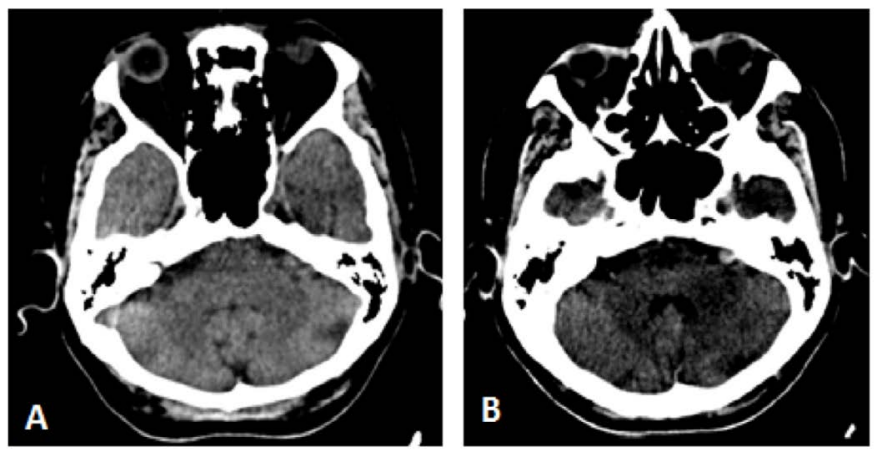

Figure 2: Axial CT scan sections prior to (A) and after contrast (B) show nodular lesion at the cerebelo-pontine angle, isoatenant to the cortex and with intense and homogeneous contrast enhancement

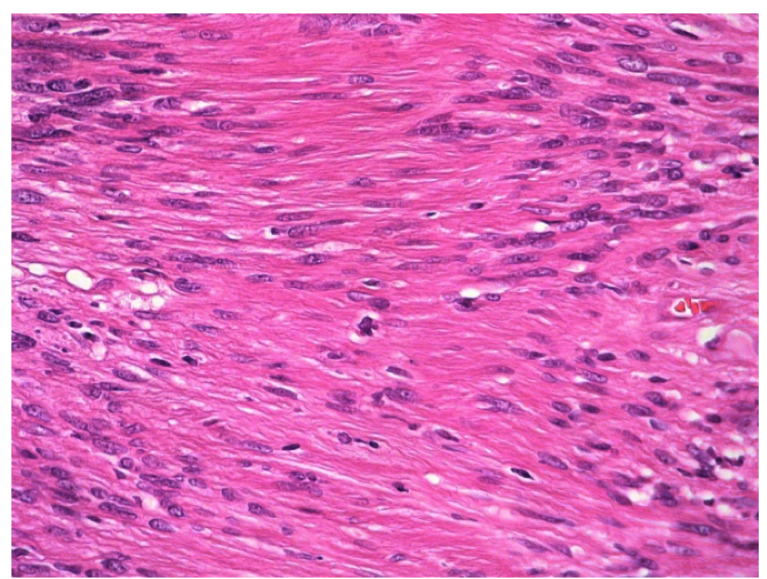

Figure 3: Histologic study of the removed tumor resulting in vestibular schwannoma. Note the presence of spindle cells in the Antoni $A$ areas, aligned in parallel rows, separated by dense stroma, and without nuclei - Verocay corpuscle (hematoxylin and eosin, 40X)

On the $15^{\text {th }}$ postoperative day the patient started complaining of headache, visual turbidity, vertigo, nausea, and high fever. After physical examination, he maintained grade IV facial paralysis, showed no signs of meningeal irritation or other neurological deficits. The operative wound had inflammatory signs and serous secretion.
During the investigation, it was collected liquor that did not show biochemical changes compatible with meningitis. CT showed failure of filling of the transverse sinus to the left sigmoid sinus, associated with postoperative changes of the mastoid (Figure 4a). MRI showed postcontrast filling failure in T1-weighted images, consistent with venous sinus thrombosis (Figure $4 \mathrm{~b}$ ).

Conservative approach was chosen for the patient, through hospitalization for venous antibiotic therapy (ceftriaxone + oxacillin), surgical wound care and local compressive dressing. After two weeks of clinical treatment, the patient presented good evolution, and was discharged.

\section{Discussion}

The postoperative of patients who underwent surgery for VS can be complicated by a number of conditions, and vascular complications have been reported in up to $7 \%$ of patients and can have devastating consequences, with significant risks of morbidity and death $[2,3]$.

Sigmoid sinus thrombosis is the least common complication of resection of the VS. Data from the literature indicate that in less than 5\% of surgeries this complication occurs, and the transverse and sigmoid sinuses on the treated side are more frequently affected, whereas the sinus contralateral to the lesion are typically spared. Keiper et al, in a study of 107 patients, found an incidence of symptomatic dural sinus thrombosis of $4.6 \%$ after translabyrinthine resections [4].

Some theories seek to explain the occurrence of dural thrombosis. The following stand out: 1) intraoperative sinus retraction; 2) dissection of the sinus during the resection of the tumor and 3) propagation of the bone wax used to control emissary veins [5]. The proximity of the lateral sinus adjacent to the mastoid cells causes infectious processes of the middle ear to extend from the mastoid to the sigmoid sinus, resulting in perivascular micro-abscesses that would involve the venous system causing septic thrombophlebitis [6]. This event occurs as complications of chronic otitis media. With the advent of the antibiotic, this complication became less frequent. It is important to remember that dehydration secondary to fluid loss and inadequate replacement or anemia secondary to excessive blood loss during a surgical procedure may have a contributory role for cerebral venous thrombosis [7].

Although more commonly asymptomatic, SST may present weeks after surgery, with various manifestations such as headache and visual turbidity. The appearance of symptoms depends on several anatomical factors, such as involvement of the dominant side, presence of sinus confluence, existence of bilateral transverse sinuses or rich
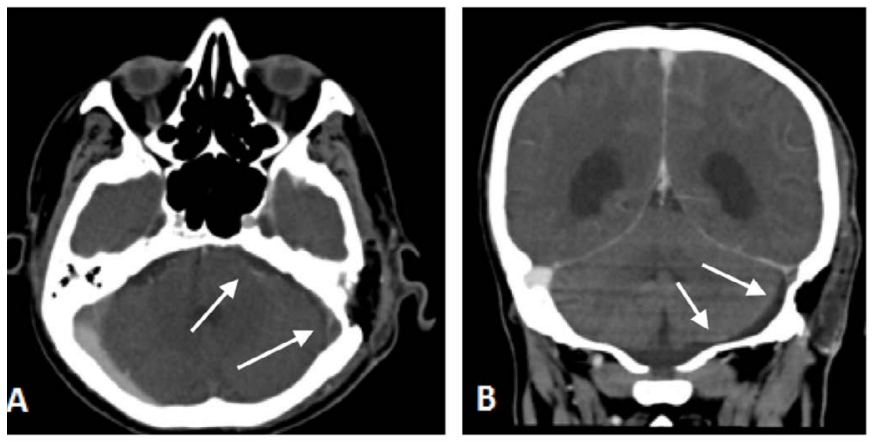

Figure 4: Post-contrast axial (A) and coronal (B) CT scans show elongated filling failure extending from the transverse sinus to the left sigmoid sinus (venous sinus thrombosis). There are also postoperative changes (signs of left mastoidectomy, with local fat filling and subcutaneous emphysema of the surgical site). 
contralateral circulation, and not only the size of the sinus itself [8]. Headache occurs in an intense, unusual manner, and may worsen with decubitus and progressive. Symptoms generally improve in days to weeks. In a retrospective study carried out by Funamura et al. during the period from 1996 to 2002, they were described as more frequent otalgia symptoms and headaches (in $60 \%$ of cases) and fever (in 100\% of cases) [9]. Symptoms such as cervical pain, isolated febrile peaks, inflammatory signs in the mastoid region were described in $20 \%$ of the cases. At physical examination, there may be increased intracranial pressure, papilledema, and retinal hemorrhage. In the case presented, as a patient presented fever and infectious changes in the operative wound, thrombophlebitis was thought to be an extension of infection of the mastoid cavity.

CT can be used as a screening tool for dural sinus thrombosis. However, MRI is a more definitive diagnostic tool. The gold standard for the detection of dural thrombosis is digital subtraction angiography, however, because it is an invasive examination, other techniques are preferred. The presence of hyperattenuating image on CT without contrast is one of the characteristic findings of thrombosis, although it is found in only $20 \%$ of the cases. On contrast CT, we can find a central triangular area that does not enhance and is delimited by the contrastenhancing dura mater, also known as the "empty delta" sign, appearing in less than $30 \%$ of the cases [10]. In relation to other tomographic findings, a retrospective study demonstrated in a series of 5 cases the presence of bone plate erosion in 4 cases. There was no significant erosion in one patient. In two patients, the thrombus was located between the sinus confluence with the jugular bulb. On the other hand, in 3 patients it was located in the right transverse and sigmoid sinus [11]. In MRI the findings vary mainly with thrombus time. In the acute phase the blood clot normally presents as an isointense image in $\mathrm{T} 1$ and hypointense in T2-weighted images, to the point that in the subacute phase thrombosis is hyperintense in T1-weighted images [12,13].

Tumors of the cerebellopontine angle are frequent, most of them represented by vestibular schwannomas and meningiomas (responding together for about $85-90 \%$ of lesions at this location). In most cases, CT and MRI are sufficient to establish the diagnosis. VSs account for $95 \%$ of cases of schwannomas, the remainder of which are represented by trigeminal nerve lesions, facial, and IX, X, XI, and XII cranial nerves [14].

Schwannoma is a benign tumor composed of Schwann cells in a collagen matrix. They may occur alone or in combination with type 2 neurofibromatosis, a condition that should be suspected when the disease is bilateral. Typical image findings of vestibular schwannomas are: expansive lesions centered on the ostium of the internal auditory canal, iso or hypointense to the cerebral cortex in T1-weighted images and discretely hyperintense in T2-weighted, typically with intense and homogeneous enhancement in T1-weighted images post- contrast [15].

In case of sinus occlusion or sacrifice of a sinus due to a more extensive approach to the base of the skull, it is important to preserve other relevant venous structures to maintain venous circulation $[4,8]$. The management of lateral sinus thrombosis is controversial due to the effects of anticoagulation. Shah et al highlights the potential complications of anticoagulation therapy including intracranial hemorrhage, drug interactions, and thrombocytopenia [16]. On the other hand, Neilan et al. [17] gathered data from 8 studies, demonstrating benefits in the use of anticoagulants, based on follow up radiological, finding in $66.7 \%$ of the patients' evidence of rechanneling of the affected sinus, in counter-departure, in patients not Subjected to therapy the percentage was $52.9 \%$. In addition to anticoagulation, treatment may include antibiotic therapy and surgical procedures (opening of the vessel to remove clots). Bradley et al emphasizes the importance of monitoring patients with MRI or CT to detect thrombus propagation [18]. In the reported clinical case, we chose conservative treatment through intravenous antibiotic therapy, with good clinical response.

\section{Conclusion}

SST is a rare postoperative complication in VS excisions and may present several clinical manifestations, making early diagnosis more difficult for physicians. The radiological findings vary according to the time of evolution, and non-invasive methods are preferable. The incidence of this disease has declined markedly with the advent of modern antibiotic therapy. However, it remains a life-threatening condition requiring a high rate of clinical suspicion for a rapid diagnosis.

\section{References}

1. House WF (1964) Acoustic neuroma. Arch Otolaryngol 80598- 80757.

2. Darrouzet V, Martel J, Enée V, Bébéar JP, Guérin J (2004) Vestibular schwannoma surgery outcomes: our multidisciplinary experience in 400 cases over 17 years. Laryngoscope 114: 681-688. [Crossref]

3. Yamakami I, Uchino Y, Kobayashi E, Yamaura A, Oka N. Removal of large acoustic neurinomas (vestibular schwannomas) by the retrosigmoid approach with no mortality and minimal morbidity (2004) J Neurol Neurosurg Psychiatry 75: 453-458. [Crossref]

4. Keiper GL, Sherman JD, Tomsick TA, Tew Jr JM (1999) Dural sinus thrombosis and pseudotumor cerebri: unexpected complications and suboccipital craniotomy and translabyrinthine craniectomy. J Neurosurg 91: 192-197. [Crossref]

5. Heman-Ackah SE, Golfinos JG, Roland Jr JT (2012) Management of surgica complications and failures in acoustic neuroma surgery. Otolaryngol Clin North Am 45: 455-470. [Crossref]

6. Penido Nde O, Toledo RN, Silveira PA, Munhoz MS, Testa JR, et al. (2007) Sigmoid sinus thrombosis associated to chronic otitis media. Braz J Otorhinolaryngol 73: 165170. [Crossref]

7. Iskandar BJ, Kapp JP (1996) Nonseptic venous occlusive disease. Neurosurg 2: 21772190.

8. Ohata K, Haque M, Morino M, Nagai K, Nishio A, et al. (1998) Occlusion of the sigmoid sinus after surgery via the presigmoidal-transpetrosal approach. $J$ Neurosurg 89: 575-584. [Crossref]

9. Funamura JL, Nguyen AT, Diaz RC (2014) Otogenic lateral sinus thrombosis: case series and controversies. Int J Pediatr Otorhinolaryngol 78: 866-870. [Crossref]

10. Poon CS, Chang JK, Swarnkar A, Johnson MH, Wasenko J (2007) Radiologic diagnosis of cerebral venous thrombosis: pictorial review. AJR Am J Roentgenol 189: S64-75. [Crossref]

11. Burakgazi G, Bayarogullari H, Oztuk F, Arli C, Motor VK, Yanmaz R, Atci N (2017) Radiological imaging of rare intracranial complications secondary to otitis media and mastoiditis. J Craniofac Surg 28: 620-624. [Crossref]

12. Gomori JM, Grossman RI, Goldberg HI, Zimmerman RA, Bilaniuk LT (1985) Intracranial hematomas: imaging by high-field MR. Radiology 157: 87-93. [Crossref]

13. Ginat DT, Meyers SP (2012) Intracranial lesions with high signal intensity on T1 weighted MR images: Differential diagnosis. Radiographics 32: 499-516. [Crossref]

14. Bonneville F, Sarrazin JL, Marsot-Dupuch K, Iffenecker C, Cordoliani YS, et al. (2001) Unusual lesions of the cerebellopontine angle: a segmental approach. Radiographics 21: 419-438. [Crossref]

15. Phillips GS, LoGerfo SE, Richardson ML, Anzai Y (2012) Interactive Web-based learning module on CT of the temporal bone: anatomy and pathology. Radiographics 32: E85-105. [Crossref]

16. Shah UK, Jubelirer TF, Fish JD, Elden LM: A caution regarding the use of lowmolecular weight heparin in pediatric otogenic lateral sinus thrombosis (2007) Int $J$ Pediatr Otorhinolaryngol 71: 347-351. [Crossref] 
Brito TP (2018) Septic thrombophlebitis of the sigmoid sinus after vestibular schwannoma resection: A case report and literature review

17. Neilan RE, Isaacson JW, Kultz JW, Lee K, Roland PS (2011) Pediatric otogenic lateral sinus thrombosis recanalization. Int J Pediatr Otorhinolaryngol 75: 850-853. [Crossref]
18. Bradley DT, Hashisaki GT, Mason JC (2002) Otogenic sigmoid sinus thrombosis: what is the role of anticoagulation? Laryngoscope 112: 1726-1729. [Crossref]

Copyright: $(02018$ Brito TP. This is an open-access article distributed under the terms of the Creative Commons Attribution License, which permits unrestricted use, distribution, and reproduction in any medium, provided the original author and source are credited. 